\title{
An Empirical Analysis of the Regional Diffusion Difference of China's Private Cars based on the G/SG Model
}

\author{
Gang Liu \\ East China University of Science and Technology \\ School of Business Administration \\ Shanghai, China \\ liugang@ecust.edu.com
}

\author{
Xianming Hong * \\ East China University of Science and Technology \\ School of Business Administration \\ Shanghai, China \\ 454479104@qq.com \\ * Corresponding Author
}

\begin{abstract}
With the markets and products evolve rapidly, enterprises have paid much attention to how products will grab the market share. The diffusion of innovation is the main mechanism of the product market's forming process, and the research on the effect of the differences between different regions to innovation diffusion will be conducive to a comprehensive understanding of the characteristic of China's regional markets and the consumer differences in different regions. Based on the G/SG model, researchers take China's private car market as an example, and analyze the diffusion of durable goods in different regions and its influence factors. The results indicate regional economy development, population mobility, education level and culture soft power have significant effects on the innovation diffusion. The significance of this paper, by studying the influence factors of innovation diffusion, is to provide the management inspiration for enterprises and government.
\end{abstract}

Keywords-Bass Model; G/SG Model; Heterogeneity; Culture Soft Power; Private Cars

\section{INTRODUCTION}

China is a diversified market with wide difference in regional economy developments, and the market structures and consumer preferences differ between the coastal and the mainland or the cities and towns, thus the model and level of innovative products' diffusion vary from region to region. Researchers conduct a study on China's situation and analyzing the diffusion behavior of innovative products in Chinese provincial market with the purpose of providing enterprises the basis to develop marketing strategies. Researchers argue that the significance of this study is that it is conducive to the discovery of market opportunities, producing a correct assessment and targeting the right market reasonably at the same time.

Early empirical research can be found in Heller and Hustad (1980), who analyzed the diffusion behavior of 15consumer durables in 27 countries, and estimated the Innovation Coefficient $\mathrm{p}$ and the Imitation coefficient $\mathrm{q}$ of the products in different countries. Gatignon et al. (1989) studied the diffusion of 6 products in 14 countries and estimated the corresponding $\mathrm{p}$ and $\mathrm{q}$ values, also they clarified that internationalization level, population mobility and women's proportion in total workforce had impacts on innovation diffusion. In Helsen et al.(1993), more macro variables were introduced in research, including population mobility, living standard, health status, international trade status, educational level and so on. On the basis of previous researches, this paper referred to some of these influence factors and provided the influences indicators of regional diffusion which are more in line with the reality condition of China.

In this paper, the regional differences of product diffusion were first studied, and then the factors that caused these differences were analyzed. Researchers mainly focused on the impact of the culture soft power and income heterogeneity on the product diffusion. This paper is organized as follows: The first section is an introduction to a sketch of the research content and related researches. Then the heterogeneity diffusion model and relevant parameter are introduced in Section 2. In Section 3, researchers put forward 4 hypotheses according to previous studies. The fourth section is the empirical analysis. The conclusion is given in the last section.

\section{INNOVATION DIFFUSION MODEL}

Bass Model is the basic model of innovation diffusion research. Early in 1969, Bass summarized the diffusion of a new product as two influence factors: the external influence $\mathrm{p}$ and the internal influence $\mathrm{q}$. In the case of firsttime purchase, Bemmaor(2002) provided an alternative derivation which is G/SG Model. Customers' adoption of new products is a stochastic process, of which the determinant is the propensities to buy. The larger the propensities to buy, the shorter the expected time to adopt, on average, early buyers exhibit larger propensities to buy than the late buyers. The basic model assumes that:

For individual customers, the adoption timing is consistent with a two-parameter shifted Gomertz distribution whose cumulative distribution function is given by

$$
\begin{gathered}
F(t \mid \eta, b)=\left(1-e^{-b t}\right) e^{\left(-\eta e^{-b t}\right)}, t>0 \\
f(t \mid \eta, b)=b e^{\left(-b t-\eta e^{-b t}\right)}\left[1+\eta\left(1-e^{-b t}\right)\right], t>0
\end{gathered}
$$

The parameter $\eta$ captures the individual-level propensity to buy. For a fixed value of $b$, the lower $\eta$ is, the lower the mean time to adoption. The parameter $b$ is a scale parameter that is constant across consumers. 
Researchers assume that the individual-level propensity to buy $\eta$ varies according to a gamma distribution across consumers.

$$
k(\eta)=\frac{1}{\beta^{\alpha} \Gamma(\alpha)} \eta^{\alpha-1} e^{-\frac{1}{\beta} \eta}, \eta, \alpha, \beta>0
$$

The gamma distribution takes a variety of shapes, depending on the value of $\alpha$. The smaller $\alpha$ is, the larger the heterogeneity. The other parameter $\beta$ of the gamma distribution is a scale parameter $($ mean $=\alpha \beta)$. It follows that the aggregate-level distribution of adoption time is

$$
\begin{gathered}
F(t)=\int_{0}^{+\infty} F(t \mid \eta, b) k(\eta) d \eta=\frac{1-e^{-b t}}{\left(1+\beta e^{-b t}\right)^{\alpha}} \\
F(t)=\frac{1-e^{-b t}}{\left(1+\beta e^{-b t}\right)^{\alpha}}
\end{gathered}
$$

When $\alpha=1, b=p+q, \quad \beta=q / p$, the model is reduced to the Bass model。

Chatterjee et al. (1990) showed that Bass model was an S-shaped curve, and when $q>p$, the larger the $q / p$ was, the more obvious the $S$ shape was, this parameter could be described as a shape parameter. According to Bass, this parameter reflects the relative importance of imitancy and innovativeness, and it determines the shape of the diffusion curve which is of great importance to the diffusion of new products. In other words, it exhibits the relative importance of the interpersonal communication and mess media to innovation diffusions. In the Van den Bulte (2004)'s study of social contagion who estimated the parameter $q / p$ utilizing the fact that income could be used to explain the purchase intention and found out that this parameter was positively associated with the difference between per capita income and Gini coefficient. In this paper, researchers conduct the fitting analysis with a specific product, and calculate the value of parameter $q / p$ and $\alpha$, the purpose of which is to analyze the influence of related factors on innovation diffusions.

\section{RESEARCH HYPOTHESIS}

In the research about the impact factors of innovation diffusion, Rogers made a comprehensive analysis and summarized the four main factors in the innovation diffusion, including innovation, time, diffusion channels and social system. Based on the previous research, the following hypotheses of several factors were put forward in this paper.

\section{A. Cultural soft power}

Van den Bulte et al. (2004) studied the impact of the indicators in national culture level on $q / p$. The result showed that different regions and different cultures have certain impact on the innovation diffusion. The concept of "Soft Power" was brought out by professor Joseph Nye[9] at Harvard University. Soft power refers primarily to the culture influence of long history and traditional advantage, which plays a catalytic role in the development of economic and the social progress.

The ability of region culture to accept new things, which is called the inclusiveness of culture, is of great importance in the diffusion of new products, for that new products can be effectively accepted only when integrated with the local culture. The level of culture reflects the driving force of the development of a region and is of significant influence to innovation diffusion. The long history, excellent traditional culture, and doctrine of the Mean in tradition culture, lead to the conservatism. At this point of view, customers are not willing to bear the risk of trial in the adoption of new products, and the innovation coefficient $p$ can be relatively lower but the internal factor $q$ stronger with strong imitative ability. Zhou etc.[10] made a comprehensive evaluation of the cultural soft power from the point of its connotation. The results were applied for analysis in this paper to study the impact of cultural soft power on innovation diffusion, and Hypothesis 1 was put forward as following.

$\mathrm{H} 1$ : The stronger the cultural soft power of a region is, the coefficient $q / p$ will be.

\section{B. Economic level}

Islam et al. (2002) collected the diffusion data of three kinds of telecommunicating devices to study the impact of GDP per capita on innovation diffusion, and the result showed that the GDP per capita was positively correlated to the speed of innovation diffusion. Cheng et al. (2010) studied the impact of economic growth on development of telecommunications, drawing a conclusion that the innovation diffusion gets faster with the development of economic. Theoretically, the higher the development level of economic is, the higher per capita income will be. The income level and its stability have a direct influence in the purchasing power of the residents as well as the consumer behavior which will become more personalized. Hence, customers become more eager to express their innovativeness when purchasing. In consequence, the more developed the economy of a region is, the smaller the value of $\mathrm{q} / \mathrm{p}$ will be. Hypothesis 2 was presented as following based on the indicator of GDP per capita.

$\mathrm{H} 2$ : The more advanced the economic level of a region is, the smaller the value of $q / p$ will be.

\section{Population mobility}

Since the reform and opening up, with the rapid economic development and urbanization process, the rural population has been transferring into cities. Hence, when researchers study the regional innovation diffusions, ignoring the population mobility is inadvisable. The mobility and migration of population have promoted the communication between different regions, also changed or improved the rural residents' consumption patterns and ideas. In other words, it has made the interpersonal communication becomes easier and provided greater opportunity for imitation behaviors. Therefore, the stronger the population mobility, the larger the value of $q / p$ will be. Researchers use the per capita consumption gasoline presented by Helsen et al. (1993) as the index of population mobility to study its influence on regional innovation diffusion and Hypothesis 3 were put forward as following.

H3: The stronger the population mobility of a region, the larger the value of $q / p$ will be. 


\section{Educational level}

According to Rogers, the early buyers who received more education than late buyers usually have a better cultural quality. The consumers with a better educational background usually have stronger cognitive competence and are less likely to be influenced by others, not to mention to imitate others. Consumers make their purchase decision according to the information they received and the specialist knowledge they have. Thus, the educational level will influence the innovation diffusion no matter internally or externally. Researchers can say that the higher the educational level is, the higher the innovativeness of the consumers and the lower the imitancy will be. In other words, as the educational level increase, the value of $q / p$ will be smaller. Researchers use the ratio between educational funds expenditure and GDP presented by Zhu et al. (2006) as the index of educational level to study its influence on regional innovation diffusion Empirical research

H4: The higher the educational level of a region is, the smaller the value of $\mathrm{q} / \mathrm{p}$ will be.

\section{THE EMPIRICAL RESEARCH}

\section{A. Data collcetion}

Due to the unavailability of the domestic car sales figures for provinces over the years, in this article, possession quantities of private cars per 100 households were adopted as the market share data. In order to ensure the authority and validity of the data, all the data were collected from official statistics, for example, China Statistical Yearbook, provincial statistics yearbooks and literature research related, etc. To study regional differences in market diffusion of the private car, relevant data of main years from 1997 to 2012 were selected for comparative analysis, and corresponding test were carried out about the impact of the regional characteristics on the diffusion of innovation.

The statistical analyze of the possession quantities of private cars per 100 households, as the household car share of the domestic regions, were conducted in accordance with the urban and rural areas respectively. Afterwards, data collected from urban and rural areas were converted to province levels according to (6)

$$
Y=\frac{x_{1} y_{1}+x_{2} y_{2}}{x_{1}+x_{2}}
$$

Where, $\mathrm{Y}, \mathrm{y}_{1}$ and $\mathrm{y}_{2}$ are the possession quantities of private cars per 100 households in provincial level, urban and rural areas, respectively; $\mathrm{x}_{1}$ and $\mathrm{x}_{2}$ are the households of the urban and rural areas in every province, respectively.

The average of the data of Year 2004 and 2005, as the middle years, were employed to ensure consistency for all the indicators in time. As for indicator data collected from other reports or documents, the year nearest to the middle year was adopted. Among the above data, GDP, gasoline consumption per capita, and educational appropriations for every region were collected from China Statistical Yearbook (Version 2005 and 2006), and the cultural soft power was from Comparative Study of Provincial Soft Power of Culture by Zhou Guofu et al. (2010).

\section{B. Model parameter Estimation}

G/SG model was applied and parameter estimation was conducted using the Nonlinear Least Square (NLS) method based on provincial data, fitting results were shown in Table 1.

TABLE I. FITTING RESULTS

\begin{tabular}{|c|c|c|c|c|c|}
\hline Region & $q / p$ & $\alpha$ & $\mathrm{R}^{2}$ & $\mathrm{~F}$ & RMSE* \\
\hline Inner & & & & & \\
\hline Mongolia & 53733194 & 0.368 & 0.983 & 819.5 & 1.59 \\
\hline Ieilongjiang & 15188196 & 0.435 & 0.989 & 1316 & 0.38 \\
\hline Tianjin & 4776240 & 0.435 & 0.997 & 5206 & 1.46 \\
\hline Hebei & 185190.6 & 0.580 & 0.979 & 641.0 & 1.68 \\
\hline Guizhou & 1409471 & 0.615 & 0.989 & 1292 & 0.49 \\
\hline Yunnan & 631.6328 & 0.888 & 0.986 & 954.4 & 0.60 \\
\hline Qinghai & 1724.458 & 0.953 & 0.974 & 517.1 & 0.39 \\
\hline Gansu & 11037.4 & 1.106 & 0.986 & 982.4 & 0.55 \\
\hline Hubei & 707.7943 & 1.289 & 0.990 & 1404 & 0.63 \\
\hline Sichuan & 87.91913 & 1.501 & 0.993 & 1998 & 0.41 \\
\hline Sinkiang & 93.90864 & 1.690 & 0.975 & 542.5 & 0.82 \\
\hline Ningxia & 67.40217 & 2.509 & 0.993 & 1992 & 0.94 \\
\hline Jilin & 14.24624 & 2.901 & 0.992 & 1773 & 0.88 \\
\hline Jiangsu & 8.177315 & 3.210 & 0.983 & 796.2 & 2.03 \\
\hline Henan & 19.10674 & 3.263 & 0.975 & 542.9 & 1.23 \\
\hline Hunan & 9.210178 & 4.123 & 0.994 & 2223 & 0.77 \\
\hline Shanxi & 4.336701 & 4.389 & 0.988 & 1161 & 1.27 \\
\hline Anhui & 8.846263 & 5.319 & 0.980 & 722.5 & 0.81 \\
\hline Jiangxi & 0.123836 & 102.4 & 0.990 & 1339 & 0.62 \\
\hline Chongqing & 0.085214 & 111.6 & 0.981 & 711.9 & 0.89 \\
\hline Tibet & 0.061884 & 145.8 & 0.983 & 826.4 & 1.40 \\
\hline Beijing & 0.015248 & 279.2 & 0.994 & 2207 & 0.74 \\
\hline Liaoning & 0.035357 & 328.6 & 0.991 & 1601 & 1.07 \\
\hline Fujian & 0.019337 & 496.1 & 0.984 & 859.1 & 1.77 \\
\hline Shandong & 0.015491 & 535.7 & 0.996 & 3298 & 0.96 \\
\hline Guangxi & 0.013282 & 759.3 & 0.992 & 1763 & 1.01 \\
\hline Guangdong & 0.005751 & 832.2 & 0.996 & 3891 & 0.99 \\
\hline Hainan & 0.004922 & 1097 & 0.984 & 860.7 & 0.99 \\
\hline Shaanxi & 0.010194 & 1137 & 0.970 & 447.2 & 1.67 \\
\hline Zhejiang & 0.004567 & 1370 & 0.992 & 1778 & 1.53 \\
\hline Shanghai & 0.002661 & 2212 & 0.981 & 738.2 & 2.07 \\
\hline
\end{tabular}

As illustrated in Table 1, the fitting results for the data of the 31 regions showed good convergence and the values of R2 were above 0.969. Additionally, F test showed good significance, which indicated favorable fitting effect. For 
Beijing, Shanghai, and Guangdong, etc, the values of $q / p$ were below 1, demonstrating that external factors have stronger impact on the diffusion of innovation than internal factors for these regions. Whereas, the values of $q / p$ were above 1 for Gansu, Hebei, Yunnan, etc., indicating that the impact of internal factors are more important for these regions.

In terms of $\alpha$, which represents the heterogeneity among consumers, the values were all below 1 for Inner Mongolia, Heilongjiang, Qinghai, Guizhou and Yunnan, etc., showing that the heterogeneity among consumers were high and the residents' income was not high, but the values for Shanghai, Zhejiang and Guangdong were large, showing relatively high income. Therefore, it could be concluded that income has certain impact on the heterogeneity among consumers in the innovation diffusion

\section{Influences of macro conditions}

The value of $\mathrm{q} / \mathrm{p}$ were taken as dependent variable, and GDP per capita, population mobility, educational level, and cultural soft power were as independent variables. Weighted least squares regression was conducted by SPSS17.0 software in order to give larger weight to the parameters with smaller errors, and a more reliable result could be obtained.

\section{(1) Metrology model}

As could be seen from previous literature research, influencing factors of the diffusion of innovation are diverse, among which four factors including cultural soft power, economic level, population mobility, and educational level were studied. The index $q / p$, relative importance of $\mathrm{q}$ (internal factor) to $p$ (external factor), was as dependent variable, and the other four indexes were as independent variables. A multiple linear regression model was established as (7).

$$
Y=\sum_{i=1}^{4} \beta_{i} X_{i}+\varepsilon, \quad \varepsilon \sim N\left(0, \sigma^{2}\right)
$$

Where, $Y$ is the value of $q / p, X_{i}$ are the four influencing factors, $\mathrm{i}$ is the coefficient of every influencing factor, $X_{1}$ is the cultural soft power, $X_{2}$ is the economic level, $X_{3}$ is the population mobility, $X_{4}$ is the educational level, and $\varepsilon$ is the stochastic error, which follows normal distribution.

(2) regression analysis

As cross-section data was used in the model, serial correlation problem did not exist in this paper. Meanwhile, the weighted least square method was adopted to eliminate the heteroscedasticity. As for the indexes selected, economic development would lead to an increase in investment in education to promote the improvement of cultural soft power, indicating that certain collinearity existed between the macro factors, but this had no impact on the validity of the regression analysis for the model. Since the dependent variable $\mathrm{q} / \mathrm{p}$ differed from the four factors in data dimension and unit, $\mathrm{Z}$ standardization was firstly conducted with the data.
Then, regression analysis was conducted based on the standardized data. The value of Sig was 0.004 with good significance. The regression results were listed in Table 2 .

In spite that the test result of the constant was not significant, there was no impact on the validity of the research. Moreover, the final regression equation could be drawn from table 2 as

$$
Y=2.087 X_{1}-2.605 X_{2}+0.893 X_{3}-1.24 X_{4}+0.115 \text {. }
$$

TABLE II. TABLE TYPE STYLE

\begin{tabular}{cccccc}
\hline & Model & B & Std.Err & T & Sig. \\
\hline (Constant) & 0.115 & 0.177 & 0.651 & 0.521 \\
Cultural soft & 2.087 & 0.658 & 3.174 & 0.004 \\
power & & & - & 0.000 \\
1 & $\begin{array}{c}\text { Economic level } \\
\text { Population } \\
\text { mobility }\end{array}$ & -2.605 & 0.619 & 4.207 & \\
& 0.893 & 0.382 & 2.339 & 0.027 \\
Educational \\
level
\end{tabular}

a. The independent variable, $\mathrm{q} / \mathrm{p}$; b. weighted least-squares regression based on RMSE.

As the results show, the hypotheses of 1-4 were all significant below the significant level (0.05), among which the indexes of cultural soft power, economic level and educational level were significant below 0.01 as well, declaring that the hypotheses proposed were all tenable. The economic meaning of this model is obvious that the four factors including cultural soft power, economic level, population mobility, and educational level have certain impact on the diffusion of innovation; the stronger the cultural soft power are, the stronger the population mobility will get, the larger $\mathrm{q} / \mathrm{p}$ is; whereas the more advanced the economic is, the higher education investment level will get, the smaller $\mathrm{q} / \mathrm{p}$ is.

\section{CONCLUSIONS}

Based on the G/SG model, this article empirically studies the relationship between the innovative diffusion coefficient $\mathrm{q} / \mathrm{p}$ and culture soft power, economic development level, population mobility and educational level. The research result verifies the four hypotheses in this article. It shows that, the stronger the culture soft power in a place, the stronger the population mobility, the bigger the coefficient; the more developed the economic level, the stronger the education investment, the smaller the coefficient.

This article not only verifies the impact of economic development level, population mobility and educational level on innovative diffusion, but also tests the impact of the culture soft power from the cultural aspect. The stronger the culture soft power, the bigger the $q / p$ coefficient, which shows the responding importance of internal and external factors. At present, researchers emphasize both hard power and soft power. Culture soft power is one of the most important impetuses of innovation, therefore, studying the impact of culture soft power on the innovative diffusion has a profound significance. 
This article provides a new angle for the market segmentation of innovative products. Based on this angle, enterprise can develop relevant marketing mix strategy to maximize its benefits. For the government, it provides the basis for relative policy setting. Traditionally, researchers segment markets according to the natural areas and macroeconomic factors, which can't provide materially guiding significance. While based on this research, from the macro perspective, researchers can not only identify market opportunity in different areas but also study innovative diffusion from the aspect of heterogeneity; from the micro perspective, researchers can study customers' preference towards new products and then formulate special strategy, such as designing products in a variety of price ranges based on their different incomes. At the same time, the government can start from the macro environment, carry forward the excellent traditional culture and promote new products through strengthening culture soft power, developing economy, increasing education investment and so on. Therefore, this article is inspirational both for enterprises and the government.

There still exist some disadvantages. For example, the result is just based on family cars, which makes it not universal. It would be much more convincing if researchers can use more types of durable consumer goods. In addition, the data about culture soft power and per capita income Gini coefficient come from somewhere else. It would be more convincing if researchers can recalculate it according to the yearbook database.

\section{REFERENCE}

[1] Heeler R M, Hustad T P. Problems in Predicting New Product Growth for Consumer Durables[J]. Management Science. 1980, 26: 1007-1020.
[2] Hubert G, Jehoshua E, Robertson Thomas S. Modeling Multinational Diffusion Patterns: an Efficient Methodology[J]. Marketing Science. 1989, 8(3): 231-247.

[3] Lee C. Determinants of national innovativeness and international market segmentation[J]. International Marketing. 1990, 7(5): 39-49.

[4] Helsen K, Jedidi K, Desarbo W S. A New Approach to Country Segmentation Utilizing Multinational Diffusion Patterns[J]. Journal of Marketing. 1993, 57: 60-71

[5] Bass F M. A New Product Growth for Model Consumer Durables[J]. Management Science. 1969, 15(5): 215-226

[6] Bemmaor A C, Lee Y. The impact of heterogeneity and illconditioning on diffusion model parameter estimates[J]. Marketing Science. 2002, 21: 209-220.

[7] Chatterjee, Rabikar, Jehoshua E. The innovation diffusion process in a heterogeneous population: A micromodeling approach[J]. Management Science. 1990, 36: 1057-1079.

[8] Van Den Bulte C, Stremersch S. Social Contagion and Income Heterogeneity in New Product Diffusion: A Meta-Analytic Test[J]. Marketing Science. 2004, 23(4): 530-544.

[9] Nye J.S .Hard and soft power[M]Beijing, Peking Unicersity Press.2005.

[10] Zhou Guofu, Wu Dandan, Comparative study of provincial soft power of culture.[J]2010,27(2):7-14

[11] Islam $\mathrm{T}$, Fiebig $\mathrm{D} \mathrm{B}$, Meade $\mathrm{N}$. Modelling multinational telecommunications demand with limited data[J]. International Journal of Forecasting. 2002(18): 605-624

[12] Cheng Pengfei,Liu Xinmei, Economy growth, substitute effect and regulation's effects on telecommunication user's growth: from innvation diffusion perspective.[J]China Soft Science, 2010(1):5359.

[13] Zhu Hengyuan et al, A case study of the effect of the dual structure upon product diffusion: taking color $\mathrm{TV}$ as examples[J]. Management World. 2006(4):66-72.

[14] Chen Changbing. Calculation of various Gini coefficients from different regions in China and Analysis using the Nonparametric model.[J]Journal of quantitative and technical economics. 2007(1):133-142 